\title{
Esperanças matemáticas dos quadrados médios: uma análise essencial
}

\author{
Mean square expected values: an essential analysis
}

\author{
Jefferson Luís Meirelles Coimbra ${ }^{1}$ Velci Queiroz de Souza ${ }^{2}$ Maurício Marini Kopp ${ }^{2}$ \\ João Gilberto Corrêa da Silva ${ }^{2}$ Antônio Costa de Oliveira ${ }^{2}$ Fernando Irajá Félix de Carvalho ${ }^{2}$
}

\section{RESUMO}

Este trabalho teve como objetivo avaliar $e$ identificar qual o tipo de soma de quadrados mais apropriada para testar hipóteses de interesse, assim como discutir alternativas mais adequadas para a solução de inconvenientes expressos por meio da análise da esperança matemática dos quadrados médios utilizados em modelos lineares mistos. A análise das esperanças matemáticas dos quadrados médios pode ser uma ferramenta de grande importância nas inferências a partir de dados experimentais, tanto incompletos (casela vazia) quanto não-balanceados. Desta forma, foram utilizados quatro exemplos, cada qual com sua peculiaridade em função do experimento ser completo ou incompleto com dados balanceados ou não-balanceados e na presença de casela vazia. O pacote estatístico SAS, versão Learning Edition, foi empregado para analisar os experimentos. $O$ resultado da análise das esperanças matemáticas dos quadrados médios indicou que a soma de quadrados do tipo I somente apresentou condições de ser utilizada em presença de dados completamente balanceados. De modo contrário, os resultados apontam que a soma de quadrados tipo III é a soma de quadrados mais apropriada no caso de dados nãobalanceados. As somas de quadrados tipo II e IV são as mais importantes no caso de caselas vazias, fato que corrobora a necessidade de avaliar sempre as esperanças matemáticas dos quadrados médios.

Palavras-chave: Avena sativa, análise de variância, modelos mistos.

\section{ABSTRACT}

This research was aimed at evaluating and identifing which type of sum of squares can be more appropriate to test hypotheses and also presenting appropriate alternatives

\begin{abstract}
to solution of problems through the analysis of mean square expected values used in the methodology of mixed linear models. The analysis of mean square expected values can be a tool of great importance in analysis of data as incomplete (empty casela) as unbalanced experiment. Therefore, four examples were used each one with its pecualiarity concerning the complete or incomplete experiment with balanced or unbalanced data and in the presence of empty casela. The SAS statistical package, version Learning Edition, was used to analyze the experiments. The result of the analysis of mean square expected values indicated that the sum of squares of the type ' $I$ ' can be used only at of condition of completely balanced data. These results indicated on the other hand, that the sum of squares of the type 'III' is the most appropriate type for unbalanced data. The sum of squares of the type 'II' and ' $I V$ ' are the most important in the case of empty caselas; fact that supports the idea of a necessity of always evaluating the mean square expected values.
\end{abstract}

Key words: Avena sativa, analysis of variance, mixed models.

\section{INTRODUÇÃO}

Quando se trabalha com ensaios que possuem números de repetições constantes juntamente com os delineamentos mais simples (inteiramente casualizado, por exemplo), a análise de experimentos fatoriais é relativamente simples, podendo ser efetuada de maneira desprovida de técnicas mais refinadas, por meio de algum pacote estatístico rotineiramente utilizado. Entretanto, quando esta condição não é

\footnotetext{
'Departamento de Fitotecnia, Universidade do Estado de Santa Catarina (UDESC). Avenida Luiz de Camões, 2090, Bairro Conta Dinheiro, 88520-000, Lages, SC, Brasil. E-mail: coimbrajefferson@cav.udesc.br.

${ }^{2}$ Departamento de Fitotecnia, Universidade Federal de Pelotas (UFPel), Campus Universitário, s/n, CP 354, 96010-900, Pelotas, RS, Brasil.

${ }^{3}$ Instituto de Física e Matemática, Departamento de Métodos Estatísticos, UFPel, Campus Universitário, s/n, CP 354, 96010-900, Pelotas, RS, Brasil.
} 
alcançada, por algum motivo alheio ao controle do pesquisador, tanto as somas de quadrados quanto as esperanças dos quadrados médios podem oferecer certas dificuldades para a escolha entre os diferentes tipos de somas de quadrados obtidas pelos vários métodos disponíveis. Assim, se há casela vazias, torna-se difícil para os estatiscistas e praticamente impossível para os pesquisadores das ciências aplicadas (IEMMA, 1995).

Os conceitos da genética biométrica raramente podem ser empregados e utilizados da maneira como se apresentam. Isso porque ambigüidades têm que ser removidas, devendo ser ainda refinadas e ajustadas para que sejam obtidas estimativas acuradas dos parâmetros genéticos pelos quais são quantificados para o uso biométrico (FALCONER \& MACKAY, 1996).

Os mesmos dados, quando analisados por diferentes tipos de soma de quadrados, podem gerar diferentes estimativas e teste $\mathrm{F}$ completamente contrastante, tanto para o fator de efeito aleatório quanto para o fator de efeito fixo (fatorial misto). $\mathrm{O}$ modelo misto ou do tipo III é aquele que apresenta tanto fatores de efeitos fixos como aleatórios, além do erro experimental (efeito aleatório) e da média da população (efeito fixo). Sendo assim, é prudente avaliar e identificar rapidamente e de maneira eficiente qual a melhor soma de quadrados a ser usada para testar as hipóteses de interesse, principalmente em modelos mistos, onde as dificuldades podem ser ainda maiores. A rigor, todos os modelos são mistos, uma vez que a média $(\mu)$ é sempre considerada fixa, e o resíduo (e) como aleatório, podendo os demais efeitos serem todos fixos, todos aleatórios ou ambos (SILVA, 1993).

Quando se trabalha com números de repetições iguais para todos os fatores e avaliados nos delineamentos mais simples, a análise destes fatores é relativamente simples e fácil. Existindo um número desigual de repetições (fatoriais desbalanceados), perde-se a ortogonalidade e os cálculos das somas de quadrados se tornam bem mais complexos (WESCHSLER, 1998). Com o avanço dos aplicativos computacionais destinados à estatística, a análise de experimentos fatoriais desbalanceados, tanto para efeitos fixos, quanto para aleatórios e mistos, tornouse facilitada (FILHO, 2002; COSTA, 2003; AGUIAR, 2003).

Embora a análise de variância (teste F) seja uma ferramenta amplamente empregada para explorar dados biológicos, poucos pacotes estatísticos habitualmente utilizados particionam a análise de variância dos quadrados médios em termos de componentes de variância. Por exemplo, em um experimento conduzido a campo sob o delineamento de blocos casualizados (KEMPTON \& FOX, 1997), o quadrado médio de blocos representa a soma de dois componentes de variância - um devido aos efeitos entre parcelas e outro devido aos efeitos entre blocos. A possibilidade de conhecer o tamanho relativo dos componentes de variância pode ser extremamente relevante para tentar compreender a variação tanto intrínseca (efeito do genótipo, por exemplo) quanto extrínseca (resíduo) dos fatores avaliados no experimento.

O presente trabalho simulou quatro tipos de somas de quadrados obtidas por meio do pacote estatístico SAS (2002) Learning Edition, em que foi provocado propositalmente tanto o desbalanceamento dos dados quanto a presença de casela vazia, gerando, deste modo, quatro exemplos que tiveram como objetivo avaliar e identificar, em cada um deles, qual será o tipo de soma de quadrados mais apropriada para testar as hipóteses de interesse. Além disso, objetivou-se apresentar alternativas para a solução destes inconvenientes por meio da análise da esperança matemática dos quadrados médios.

\section{MATERIAL E MÉTODOS}

Este trabalho foi realizado utilizando cinco genótipos de aveia em três ambientes diferentes, sendo que o experimento foi conduzido segundo o delineamento inteiramente casualizado. Os dados foram selecionados por não apresentarem significância do efeito da interação genótipo $\mathrm{x}$ ambiente $(\mathrm{G} \times \mathrm{A})$, permitindo, com isso, simplificar tanto a programação dos procedimentos do SAS quanto a interpretação dos resultados obtidos. Para a realização das análises, foram empregados os procedimentos GLM e Mixed, sendo que os efeitos de genótipos foram considerados aleatórios para ambos os procedimentos, para todas as análises realizadas. Os efeitos de ambientes foram considerados como fixos em ambos os procedimentos.

Para a verificação da adequação do procedimento para cada análise gerada, foram obtidos os quatro tipos de soma de quadrados, com suas respectivas esperanças matemáticas, sendo considerado o melhor tipo aquele que revelou a menor diferença entre os coeficientes dos componentes da variância (FILHO, 2003). O estudo da formulação e a parametrização das esperanças dos quadrados médios para efeitos aleatórios em modelos mistos revelam uma controvérsia na literatura. Confusão surge quanto a aceitar ou não soma igual a zero para o termo que envolve efeito fixo. CORNFIELD \& TUKEY (1956) e WINER (1971) assumem que a soma deva ser igualada a zero; de modo contrário, SEARLE(1971) e HOCKING 
(1985) (incluindo o PROC GLM) não reconhecem esta condição. Suposições diferentes podem gerar esperanças matemáticas diferentes para certos termos incluídos no modelo e, consequentemente, valores desiguais para o teste $\mathrm{F}$.

Os dados primeiramente foram analisados, de forma balanceada nos procedimentos GLM e Mixed, do programa estatístico SAS (2002) Learning Edition, nos quais foram identificados quatro tipos de somas de quadrados (Tipo I, II, III e IV). Os dados com as estruturas dispostas aleatoriamente, de forma a causar um desbalanceamento, como pode ser observado por meio da tabela 1 , foram também submetidos aos procedimentos GLM (General Linear Models) e Mixed, obtendo os quatro tipos de soma de quadrados. Os mesmos dados foram manipulados de forma aleatória, de modo a obter caselas vazias, com dados balanceados e com dados não balanceados (Tabela 1), procedimento com o qual também foram obtidos quatro tipos de soma de quadrados (conforme nomenclatura do SAS: ss 1, ss 2, ss3 e ss 4 ).

As estimativas dos componentes da variância foram obtidas por REML, implementado no SAS. A preferência por este método decorre de suas

Tabela 1 - Croqui representativo dos exemplos com dados balanceados, não balanceados, dados balanceados com casela vazia e dados nãobalanceados com casela vazia. Pelotas, 2004.

\begin{tabular}{|c|c|c|c|c|c|c|c|c|c|}
\hline \multicolumn{10}{|c|}{ Balanceado } \\
\hline \multirow{3}{*}{ Genótipo } & \multicolumn{9}{|c|}{ Ambiente } \\
\hline & \multicolumn{3}{|c|}{1} & \multicolumn{3}{|c|}{2} & \multicolumn{3}{|c|}{3} \\
\hline & 1 & 2 & 3 & 1 & 2 & 3 & 1 & 2 & 3 \\
\hline 1 & y111 & y112 & y113 & y121 & y122 & y123 & y131 & y132 & y133 \\
\hline 2 & y211 & y212 & y213 & y221 & y222 & y223 & y231 & y232 & y233 \\
\hline 3 & y311 & y312 & y313 & y321 & y322 & y323 & y331 & y332 & y333 \\
\hline 4 & y411 & y412 & y413 & $\mathrm{y} 421$ & y422 & y423 & y431 & $\mathrm{y} 432$ & $\mathrm{y} 433$ \\
\hline 5 & y511 & y512 & y513 & y521 & y522 & y523 & y531 & y532 & y533 \\
\hline \multicolumn{10}{|c|}{ Não-balanceado } \\
\hline & \multicolumn{9}{|c|}{ Ambiente } \\
\hline \multirow[t]{2}{*}{ Genótipo } & \multicolumn{3}{|c|}{1} & \multicolumn{3}{|c|}{2} & \multicolumn{3}{|c|}{3} \\
\hline & 1 & 2 & 3 & 1 & 2 & 3 & 1 & 2 & 3 \\
\hline 1 & . & y112 & y113 & . & y122 & y123 & . & y132 & y133 \\
\hline 2 & y211 & y212 & y213 & Y221 & y222 & y223 & y231 & y232 & y233 \\
\hline 3 & y311 & y312 & y313 & Y321 & y322 & y323 & y331 & y332 & y333 \\
\hline 4 & y411 & y412 & y413 & Y421 & y422 & y423 & y431 & y432 & $\mathrm{y} 433$ \\
\hline 5 & y511 & y512 & y513 & Y521 & y522 & y523 & y531 & y532 & y533 \\
\hline \multicolumn{10}{|c|}{ Balanceado com casela vazia } \\
\hline \multirow{3}{*}{ Genótipo } & \multicolumn{9}{|c|}{ Ambiente } \\
\hline & \multicolumn{3}{|c|}{1} & \multicolumn{3}{|c|}{2} & \multicolumn{3}{|c|}{3} \\
\hline & 1 & 2 & 3 & 1 & 2 & 3 & 1 & 2 & 3 \\
\hline 1 & . & . & . & y121 & y122 & y123 & y131 & y132 & y133 \\
\hline 2 & y211 & y212 & y213 & y221 & y222 & y223 & y231 & y232 & y233 \\
\hline 3 & y311 & y312 & y313 & y321 & y322 & y323 & y331 & y332 & y333 \\
\hline 4 & y411 & y412 & y413 & y421 & y422 & y423 & y431 & y432 & $\mathrm{y} 433$ \\
\hline 5 & y511 & y512 & y513 & y521 & y522 & y523 & y531 & y532 & y533 \\
\hline \multicolumn{10}{|c|}{ Não-balanceado com casela vazia } \\
\hline \multirow{3}{*}{ Genótipo } & \multicolumn{9}{|c|}{ Ambiente } \\
\hline & \multicolumn{3}{|c|}{1} & \multicolumn{3}{|c|}{2} & \multicolumn{3}{|c|}{3} \\
\hline & 1 & 2 & 3 & 1 & 2 & 3 & 1 & 2 & 3 \\
\hline 1 & . & y112 & y113 & . & y122 & y123 & . & y132 & y133 \\
\hline 2 & . & . & . & y221 & y222 & y223 & y231 & y232 & y233 \\
\hline 3 & y311 & y312 & y313 & y321 & y322 & y323 & y331 & y332 & y333 \\
\hline 4 & y411 & y412 & y413 & y421 & $\mathrm{y} 422$ & y423 & $\mathrm{y} 431$ & y432 & $\mathrm{y} 433$ \\
\hline 5 & y511 & y512 & y513 & y521 & y522 & y523 & y531 & y532 & y533 \\
\hline
\end{tabular}

Ciência Rural, v.36, n.6, nov-dez, 2006. 
propriedades estatísticas, superiores às propriedades dos estimadores de quadrados mínimos e de máxima verossimilhança (SEARLE et al., 1992).

De acordo com SPEED \& HOCKING (1976)

e GOODNIGHT (1980), os quatro tipos de somas de quadrados são chamados de tipo I, tipo II, tipo III e tipo IV, e podem ser obtidos pela diferença entre a redução na soma de quadrados, por causa do ajuste de dois modelos diferentes. Nas somas de quadrados do tipo I, ou seqüencial, cada efeito é ajustado somente para os efeitos precedentes no modelo, enquanto que, nas somas de quadrados tipo II ou parciais, cada fator é ajustado para todos os outros apropriados. Nesse sentido, o termo "apropriados" significa que essas somas de quadrados são para algum fator ajustado para todos os outros, exceto para interação que envolve estes fatores e fatores hierárquicos. Já as somas de quadrados do tipo III ou parcial, referem-se ao ajuste de cada causa de variação para todos os efeitos remanescentes do modelo, inclusive para as interações que envolvem essa causa de variação, sob restrição paramétrica do tipo soma dos efeitos igual a zero (FREUND \& LITTEL, 1981). As funções estimáveis do tipo IV não são geralmente únicas, quando há caselas vazias, pois elas dependem da quantidade e da posição de tais caselas. Portanto, havendo casela vazia, a interpretação da soma de quadrados tipo IV não pode ser feita sem antes examinar as funções estimáveis envolvidas, para saber qual delas está sendo gerada e testada (COSTA, 2000, NESI, 2002).

Uma adequada caracterização tanto do efeito do fator quanto da avaliação e da escolha das esperanças matemáticas dos quadrados médios é de suma importância, por ter implicação direta nas inferências que serão derivadas do experimento, particularmente quanto ao âmbito de sua validade com respeito ao tipo de fator. No caso de fator fixo, as inferências limitam-se aos níveis avaliados no experimento, enquanto que, para o fator aleatório, estende-se a população de níveis, da qual os níveis incluídos no experimento constituem uma amostra aleatória. A partir disso, a caracterização do fator e sua conseqüente classificação como fixo ou aleatório é essencialmente determinada pelo objetivo do experimento.

As linhas de comando da programação SAS empregadas foram as seguintes: proc glm;

class genot amb; model $y=$ genot $\mid a m b / s s 1$ ss 2 ss 3 ss4; random genot genot*amb/test;

run; proc mixed ord;

class genot amb; model $y=a m b ;$ random genot genot*amb/solution;

run;

\section{RESULTADOS E DISCUSSÃO}

Observando a tabela 2, pode ser constatado que, todos os diferentes tipos de soma de quadrados (tipo I, II, III e IV) são idênticos no caso de existir o balanceamento dos dados, mostrando que a hipótese testada apresentou mesmo resultado em ambos os tipos de análise. Portanto, especificamente, o emprego tanto do procedimento GLM quanto do procedimento Mixed é equivalente nesta situação. A análise de variância realizada pelo proc glm apontou diferenças $(\mathrm{P}<0,05)$ significativas para todas as fontes de variação incluídas no modelo, excetuando o efeito da interação G x A. No entanto, o proc glm utiliza como denominador para a confecção dos testes na análise de variância, para todos os efeitos, o quadrado médio do resíduo (29.417). Como, neste caso, existem tanto fatores de efeito fixo (A) quanto aleatório (G), o exemplo nitidamente se caracteriza por um esquema fatorial de efeito misto ou do tipo III, porém balanceado. Desse modo, o quadrado médio apropriado para testar os efeitos tanto do fator fixo quanto do fator aleatório deve ser aquele quadrado médio associado ao efeito da interação entre os fatores (GxA).

Esta inferência pode ser obtida pela simples análise das esperanças matemáticas dos quadrados médios $(\mathrm{E}(\mathrm{QM}))$, que correspondem ao que se espera que ocorra em média com a variável resposta em questão (RAMALHO et al., 2000). Igualando-se as esperanças matemáticas do quadrado médio do fator genótipo com as esperanças dos quadrados médios do efeito da interação $\mathrm{G}$ x A, pode ser observado que sobra somente um componente de variância, fornecendo assim, um teste exato dos efeitos do fator avaliado. Além disto, a análise das esperanças matemáticas dos quadrados médios, ou componentes de variância, possibilita conhecer a estrutura genética das populações e estimar os parâmetros relacionados com os processos seletivos como coeficientes de herdabilidades e grau médio de dominância, por exemplo, (JUNIOR, 1989; LITTEL et al., 1996). Assim, a importância desta metodologia para o melhoramento de plantas é muito grande, pois permite estudar os processos seletivos com uma base científica.

Por definição, componentes de variância são variâncias associadas aos efeitos aleatórios de um modelo matemático (BARBIN, 1993). As estimativas dos componentes da variância ou da variabilidade existente nas populações e, mais ainda, de quanto desta 
Tabela 2 - Resumo da análise de variância para a variável rendimento de grãos em aveia (kg ha $\left.{ }^{-1}\right)$. Pelotas, 2005.

\begin{tabular}{|c|c|c|c|c|c|c|c|c|c|}
\hline \multirow{3}{*}{ FV } & \multirow{3}{*}{ GL } & \multicolumn{6}{|c|}{ Balanceados } & & \\
\hline & & \multicolumn{2}{|c|}{ Tipo I } & \multicolumn{2}{|c|}{ Tipo II } & \multicolumn{2}{|c|}{ Tipo III } & \multicolumn{2}{|c|}{ Tipo IV } \\
\hline & & SQ & QM & SQ & QM & SQ & $\mathrm{QM}$ & SQ & QM \\
\hline Genótipo (G) & 3 & 268.500 & $89.500^{*}$ & 268.500 & $89.500^{*}$ & 268.500 & $89.500^{*}$ & 268.500 & $89.500^{*}$ \\
\hline Ambiente (A) & 2 & 513.980 & $256.990^{*}$ & 513.980 & $256.990^{*}$ & 513.980 & $256.990^{*}$ & 513.980 & $256.990^{*}$ \\
\hline $\mathrm{G}^{*} \mathrm{~A}$ & 6 & 224.865 & $37.477^{\mathrm{ns}}$ & 224.865 & $37.477^{\mathrm{ns}}$ & 224.865 & $37.477^{\mathrm{ns}}$ & 224.865 & $37.477^{\mathrm{ns}}$ \\
\hline Resíduo & 24 & 706.008 & 29.417 & 706.008 & 29.417 & 706.008 & 29.417 & 706.008 & 29.417 \\
\hline Total & 35 & 1.713 .354 & & 1.713 .354 & & 1.713 .354 & & 1.713 .354 & \\
\hline \multicolumn{10}{|c|}{ Não-balanceados } \\
\hline Genótipo (G) & 3 & 267.317 & $89.106^{\mathrm{ns}}$ & 271.336 & $90.445^{\mathrm{ns}}$ & 268.945 & $89.648^{\mathrm{ns}}$ & 268.945 & $89.648^{\mathrm{ns}}$ \\
\hline Ambiente (A) & 2 & 517.576 & $258.788^{*}$ & 517.576 & $258.788^{*}$ & 498.266 & $249.133^{*}$ & 498.266 & $249.132^{*}$ \\
\hline $\mathrm{G}^{*} \mathrm{~A}$ & 6 & 217.642 & $36.273^{\mathrm{ns}}$ & 217.642 & $36.273^{\mathrm{ns}}$ & 217.642 & $36.274^{\mathrm{ns}}$ & 217.643 & $36.274^{\mathrm{ns}}$ \\
\hline Resíduo & 22 & 705.071 & 32.049 & 705.071 & 32.049 & 705.071 & 32.049 & 705.071 & 32.049 \\
\hline Total & 33 & 1.707 .606 & & 1.707 .606 & & 1.707 .606 & & 1.707 .606 & \\
\hline \multicolumn{10}{|c|}{ Balanceados casela vazia } \\
\hline Genótipo (G) & 3 & 202.710 & $67.570^{\mathrm{ns}}$ & 211.335 & $70.445^{\mathrm{ns}}$ & 211.335 & $70.445^{\mathrm{ns}}$ & 205.746 & $68.582^{\mathrm{ns}}$ \\
\hline Ambiente (A) & 2 & 409.887 & $204.944^{*}$ & 409.887 & $204.944^{*}$ & 409.887 & $204.944^{*}$ & 4.177.223 & $208.612^{*}$ \\
\hline $\mathrm{G}^{*} \mathrm{~A}$ & 5 & 185.310 & $37.062^{\mathrm{ns}}$ & 185.310 & $37.062^{\mathrm{ns}}$ & 185.310 & $37.062^{\mathrm{ns}}$ & 185.310 & $37.062^{\mathrm{ns}}$ \\
\hline Resíduo & 22 & 674.616 & 30.664 & 674.616 & 30.664 & 674.616 & 30.664 & 674.616 & 30.664 \\
\hline Total & 32 & 1.472 .524 & & 1.472 .524 & & 1.472 .524 & & 1.472 .524 & \\
\hline \multicolumn{10}{|c|}{ Não-balanceados casela vazia } \\
\hline Genótipo (G) & 3 & 208.854 & $69.618^{\mathrm{ns}}$ & 217.517 & $72.506^{\mathrm{ns}}$ & 215.930 & $71.977^{\mathrm{ns}}$ & 211801 & $70.600^{\mathrm{ns}}$ \\
\hline Ambiente (A) & 2 & 375.591 & $187.795^{*}$ & 375.591 & $187.795^{*}$ & 333.161 & $166.580^{*}$ & 334227 & $167.113^{*}$ \\
\hline $\mathrm{G}^{*} \mathrm{~A}$ & 5 & 190.873 & $38.175^{\mathrm{ns}}$ & 190.872 & $38.175^{\mathrm{ns}}$ & 190.873 & $38.175^{\mathrm{ns}}$ & 190873 & $38.175^{\mathrm{ns}}$ \\
\hline Resíduo & 19 & 662.412 & 34.864 & 662.412 & 34.864 & 662.412 & 34.864 & 662.412 & 34.864 \\
\hline Total & 29 & 1.437 .729 & & & & & & & \\
\hline
\end{tabular}

variabilidade é devida a diferenças genéticas são de fundamental importância em qualquer programa de melhoramento, pois permitem conhecer tanto o controle genético do caráter quanto o potencial da população para seleção (RAMALHO et al., 2004).

O programa estatístico SAS possibilita incluir a opção Test na programação do proc glm. Utilizando esta opção, pode ser observado que os quadrados médios empregados como denominadores coincidem literalmente com os quadrados médios apropriados. No entanto, o proc glm não reconhece os efeitos do fator aleatório como aleatórios, e sim como fixos, motivo pelo qual o teste F realizado não é o mais indicado.

Análises de variância obtidas por meio do procedimento mixed, inseridas na tabela 3 , descrevem as estimativas dos componentes de variância dos fatores de efeito aleatórios ( $\mathrm{G}$ e $\mathrm{G}$ x A) e o efeito do fator fixo (A). É de grande relevância observar que o procedimento mixed, ao contrário do $\mathrm{glm}$, reconhece, os efeitos aleatórios como aleatórios. Contudo, para este caso específico, onde os dados são balanceados, a variância do resíduo obtida tanto pelo proc mixed quanto pelo proc glm, após a indispensável inclusão da opção Test, fornece a mesma estimativa para este componente da variação (Tabela 2).

Em relação ao primeiro exemplo, foram eliminadas aleatoriamente algumas observações com intuito de tornar os dados não-balanceados (Tabela 2). Analisando as somas de quadrados inseridas na tabela 2 , pode ser observado que os quatro tipos de soma de quadrados diferem, excetuando as somas de quadrados tipo III e tipo IV; pois estas somas de quadrados não diferiram em virtude da ausência de casela vazia (os exemplos 3 e 4 ilustram o fato a seguir). Fatoriais com casela vazia, isto é, com um ou mais tratamentos sem nenhuma observação, são relatados na literatura como sendo um tipo de análise de grande complexidade (FILHO, 2002). Da mesma forma do primeiro exemplo, os testes para os efeitos inseridos no modelo não são apropriados. Este fato torna necessário um exame meticuloso das esperanças matemáticas dos quadrados médios para cada um dos tipos diferentes de soma de quadrados I, II, III e IV.

Analisando o fator de tratamento genótipo, pode ser observado facilmente que a soma de quadrado para os dados não-balanceados do tipo I não é apropriada (Tabela 4), pois este termo não se ajusta 
Tabela 3 - Estimativas dos componentes de variâncias (CV) para os efeitos aleatórios (genótipos e interação genótipo x ambiente) e teste F para o fator considerado de efeito fixo (ambiente) realizadas por meio do procedimento Mixed para os quatro exemplos abordados neste estudo (dados balanceados, não balanceados, balanceados com casela vazia e não-balanceados com casela vazia). Pelotas, 2005.

\begin{tabular}{|c|c|c|c|}
\hline \multicolumn{4}{|c|}{ Dados balanceados } \\
\hline Efeito aleatório & Estimativas (CV) & Efeito fixo & $\mathrm{F}$ (p-valor) \\
\hline Genótipos(G) & 5.780 & Ambiente (A) & $6,86(0,0086)$ \\
\hline $\mathrm{G}^{*} \mathrm{~A}$ & 2.687 & & \\
\hline Resíduo & 29.417 & & \\
\hline \multicolumn{4}{|c|}{ Dados não balanceados } \\
\hline Genótipos(G) & 6.094 & Ambiente (A) & $6,85(0,0086)$ \\
\hline $\mathrm{G}^{*} \mathrm{~A}$ & 2.005 & & \\
\hline Resíduo & 31.671 & & \\
\hline \multicolumn{4}{|c|}{ Dados balanceados com casela vazia } \\
\hline Genótipos(G) & 3.761 & Ambiente (A) & $3,77(0,0401)$ \\
\hline $\mathrm{G}^{*} \mathrm{~A}$ & 6.738 & & \\
\hline Resíduo & 32.614 & & \\
\hline \multicolumn{4}{|c|}{ Dados não balanceados com casela vazia } \\
\hline Genótipos(G) & $1,24^{-13}$ & Ambiente (A) & $3,66(0,0357)$ \\
\hline $\mathrm{G}^{*} \mathrm{~A}$ & 0 & & \\
\hline Resíduo & 105.805 & & \\
\hline
\end{tabular}

para os demais fatores inseridos no modelo. No entanto, as somas de quadrados do tipo III e IV são equivalentes e também são os tipos de somas de quadrados mais adequadas para este caso. Pois, a relação entre $\mathrm{s}($ erro) $+2,80 \mathrm{~s}\left(\mathrm{G}^{*} \mathrm{~A}\right)+8,4 \mathrm{~s}(\mathrm{~A})$ e $\mathrm{s}($ erro $)+2,8081 \mathrm{~s}\left(\mathrm{G}^{*} \mathrm{~A}\right)$ fornece um teste de melhor aproximação em relação aos tipos I e II. Tal fato ocorre porque a diferença entre 2,8 e 2,8081 (Tipo III) é menor em valor absoluto que a razão entre 2,8384 e 2,8081 (Tipo II); sendo assim, os pesquisadores devem optar pela soma de quadrados tipo III após o exame das esperanças matemáticas dos quadrados médios, para este caso específico. Cabe ser ressaltado que os resultados encontrados neste trabalho estão de acordo com FILHO (2003).

Outro fato relevante que deve ser observado é a respeito do incremento (em torno de 10\%), em termos do valor numérico, que houve no quadrado médio do resíduo, comparativamente, entre os dados balanceados e não balanceados. Para CARDELLINO \& SIEWERDT (1992), quando um experimento é conduzido, dois são os objetivos maiores a serem alcançados. O primeiro consiste em obter uma estimativa do erro experimental e o segundo em testar a significância dos contrastes. De acordo com estes autores, ainda é importante a estimativa correta do erro experimental, pois este é utilizado em qualquer procedimento posterior de comparação de médias. Tal fato elucida a importância prática e aplicada da escolha correta entre os diferentes tipos de soma de quadrados.
Observando o valor de probabilidade para o teste de efeito fixo obtido pelo proc $\mathrm{glm}$, depois das devidas correções $(0,0271)$, é aparente que este é aproximadamente cinco vezes maior que o fornecido pelo proc mixed $(0,0086)$.

As esperanças matemáticas dos quadrados médios apresentados na tabela 4, para os dados balanceados com casela vazia, revelam que, novamente, existem diferenças entre os diferentes tipos de somas de quadrados. Uma vez que, além da perda de casela(s) vazia(s), o proc glm ignora o efeito aleatório, uma alternativa para contornar este inconveniente pode ser obtida por meio do proc mixed, o qual fornece estimativas de médias ajustadas (lsmeans) mesmo na ocorrência de caselas vazias. Outro fato que deve ser novamente observado são os incrementos que ocorreram concomitantemente nas estimativas dos quadrados médios do erro. Isso sugere a importância de conduzir ensaios da melhor maneira possível, pois, à medida que foram eliminadas ou excluídas observações do ensaio, ocorreu um aumento nas estimativas do quadrado médio do erro ou do resíduo.

Estudando o efeito do fator aleatório (G), pode ser observado que a soma de quadrados do tipo I é a menos apropriada, comparativamente com as demais somas de quadrados estimados (Tabela 2), pois não está ajustada para o fator genótipo. No entanto, a soma de quadrados do tipo IV é a mais apropriada para este caso tanto para o fator genótipo quanto para o fator ambiente. Por exemplo, a relação entre s(erro) + 
Tabela 4 - Resumo das esperanças matemáticas dos quadrados médios. Pelotas, 2005.

\begin{tabular}{|c|c|c|}
\hline \multicolumn{3}{|c|}{ Balanceados } \\
\hline Tipo & Fonte Variação & Esperanças matemáticas \\
\hline \multirow{3}{*}{ I } & Genótipo $(\mathrm{G})$ & $\sigma($ erro $)+3 \sigma\left(\mathrm{G}^{*} \mathrm{~A}\right)+9 \sigma(\mathrm{G})$ \\
\hline & Ambiente (A) & $\sigma($ erro $)+3 \sigma\left(\mathrm{G}^{*} \mathrm{~A}\right)+\mathrm{Q}(\mathrm{A})^{(1)}$ \\
\hline & $\mathrm{G}^{*} \mathrm{~A}$ & $\sigma($ erro $)+3 \sigma\left(\mathrm{G}^{*} \mathrm{~A}\right)$ \\
\hline \multirow{3}{*}{ II } & Genótipo (G) & $\sigma($ erro $)+3 \sigma\left(\mathrm{G}^{*} \mathrm{~A}\right)+9 \sigma(\mathrm{G})$ \\
\hline & Ambiente (A) & $\sigma($ erro $)+3 \sigma\left(\mathrm{G}^{*} \mathrm{~A}\right)+\mathrm{Q}(\mathrm{A})$ \\
\hline & $\mathrm{G}^{*} \mathrm{~A}$ & $\sigma($ erro $)+3 \sigma\left(\mathrm{G}^{*} \mathrm{~A}\right)$ \\
\hline \multirow{3}{*}{ III } & Genótipo (G) & $\sigma($ erro $)+3 \sigma\left(\mathrm{G}^{*} \mathrm{~A}\right)+9 \sigma(\mathrm{G})$ \\
\hline & Ambiente (A) & $\sigma($ erro $)+3 \sigma\left(\mathrm{G}^{*} \mathrm{~A}\right)+\mathrm{Q}(\mathrm{A})$ \\
\hline & $\mathrm{G}^{*} \mathrm{~A}$ & $\sigma($ erro $)+3 \sigma\left(\mathrm{G}^{*} \mathrm{~A}\right)$ \\
\hline \multirow{3}{*}{ IV } & Genótipo (G) & $\sigma($ erro $)+3 \sigma\left(\mathrm{G}^{*} \mathrm{~A}\right)+9 \sigma(\mathrm{G})$ \\
\hline & Ambiente (A) & $\sigma($ erro $)+3 \sigma\left(\mathrm{G}^{*} \mathrm{~A}\right)+\mathrm{Q}(\mathrm{A})$ \\
\hline & $\mathrm{G}^{*} \mathrm{~A}$ & $\sigma($ erro $)+3 \sigma\left(\mathrm{G}^{*} \mathrm{~A}\right)$ \\
\hline \multicolumn{3}{|c|}{ Balanceados casela vazia } \\
\hline \multirow{3}{*}{ I } & Genótipo (G) & $\sigma($ erro $)+2,5245 \sigma\left(\mathrm{G}^{*} \mathrm{~A}\right)+6,4359 \sigma(\mathrm{G})+\mathrm{Q}(\mathrm{A})$ \\
\hline & Ambiente (A) & $\sigma($ erro $)+2,3491 \sigma\left(\mathrm{G}^{*} \mathrm{~A}\right)+\mathrm{Q}(\mathrm{A})$ \\
\hline & $\mathrm{G}^{*} \mathrm{~A}$ & $\sigma($ erro $)+2,238 \sigma\left(\mathrm{G}^{*} \mathrm{~A}\right)$ \\
\hline \multirow{3}{*}{ II } & Genótipo (G) & $\sigma($ erro $)+2,5081 \sigma\left(\mathrm{G}^{*} \mathrm{~A}\right)+6,2381 \sigma(\mathrm{G})$ \\
\hline & Ambiente (A) & $\sigma($ erro $)+2,3491 \sigma\left(\mathrm{G}^{*} \mathrm{~A}\right)+\mathrm{Q}(\mathrm{A})$ \\
\hline & $\mathrm{G}^{*} \mathrm{~A}$ & $\sigma($ erro $)+2,238 \sigma\left(\mathrm{G}^{*} \mathrm{~A}\right)$ \\
\hline \multirow{3}{*}{ III } & Genótipo (G) & $\sigma($ erro $)+2,1871 \sigma\left(\mathrm{G}^{*} \mathrm{~A}\right)+5,792 \sigma(\mathrm{G})$ \\
\hline & Ambiente (A) & $\sigma($ erro $)+2,1921 \sigma\left(\mathrm{G}^{*} \mathrm{~A}\right)+\mathrm{Q}(\mathrm{A})$ \\
\hline & $\mathrm{G}^{*} \mathrm{~A}$ & $\sigma(\mathrm{erro})+2,238 \sigma\left(\mathrm{G}^{*} \mathrm{~A}\right)$ \\
\hline \multirow{3}{*}{ IV } & Genótipo (G) & $\sigma($ erro $)+2,2063 \sigma\left(\mathrm{G}^{*} \mathrm{~A}\right)+5,6 \sigma(\mathrm{G})$ \\
\hline & Ambiente (A) & $\sigma($ erro $)+2,2448 \sigma\left(\mathrm{G}^{*} \mathrm{~A}\right)+\mathrm{Q}(\mathrm{A})$ \\
\hline & $\mathrm{G}^{*} \mathrm{~A}$ & $\sigma($ erro $)+2,238 \sigma\left(\mathrm{G}^{*} \mathrm{~A}\right)$ \\
\hline \multicolumn{3}{|c|}{ Não-balanceados } \\
\hline \multirow{3}{*}{ I } & Genótipo (G) & $\sigma($ erro $)+2,8487 \sigma\left(\mathrm{G}^{*} \mathrm{~A}\right)+0,0252 \sigma(\mathrm{A})+8,4706 \sigma(\mathrm{G})$ \\
\hline & Ambiente (A) & $\sigma($ erro $)+2,8615 \sigma\left(\mathrm{G}^{*} \mathrm{~A}\right)+11,286 \sigma(\mathrm{A})$ \\
\hline & $\mathrm{G}^{*} \mathrm{~A}$ & $\sigma($ erro $)+2,8081 \sigma\left(\mathrm{G}^{*} \mathrm{~A}\right)$ \\
\hline \multirow{3}{*}{ II } & Genótipo (G) & $\sigma($ erro $)+2,8384 \sigma\left(\mathrm{G}^{*} \mathrm{~A}\right)+8,4545 \sigma(\mathrm{G})$ \\
\hline & Ambiente (A) & $\sigma($ erro $)+2,8615 \sigma\left(\mathrm{G}^{*} \mathrm{~A}\right)+11,286 \sigma(\mathrm{A})$ \\
\hline & $\mathrm{G}^{*} \mathrm{~A}$ & $\sigma($ erro $)+2,8081 \sigma\left(\mathrm{G}^{*} \mathrm{~A}\right)$ \\
\hline \multirow{3}{*}{ III } & Genótipo (G) & $\sigma($ erro $)+2,8000 \sigma\left(\mathrm{G}^{*} \mathrm{~A}\right)+8,400 \sigma(\mathrm{G})$ \\
\hline & Ambiente (A) & $\sigma($ erro $)+2,7733 \sigma\left(\mathrm{G}^{*} \mathrm{~A}\right)+11,093 \sigma(\mathrm{A})$ \\
\hline & $\mathrm{G}^{*} \mathrm{~A}$ & $\sigma($ erro $)+2,8081 \sigma\left(\mathrm{G}^{*} \mathrm{~A}\right)$ \\
\hline \multirow{3}{*}{ IV } & Genótipo (G) & $\sigma($ erro $)+2,8000 \sigma\left(\mathrm{G}^{*} \mathrm{~A}\right)+8,400 \sigma(\mathrm{G})$ \\
\hline & Ambiente (A) & $\sigma($ erro $)+2,7733 \sigma\left(\mathrm{G}^{*} \mathrm{~A}\right)+11,093 \sigma(\mathrm{A})$ \\
\hline & $\mathrm{G}^{*} \mathrm{~A}$ & $\sigma($ erro $)+2,8081 \sigma\left(\mathrm{G}^{*} \mathrm{~A}\right)$ \\
\hline \multirow{4}{*}{ I } & Não-bc & vazia \\
\hline & Genótipo (G) & $\sigma($ erro $)+2,4581 \sigma\left(\mathrm{G}^{*} \mathrm{~A}\right)+3,1282 \sigma(\mathrm{A})+7,6344 \sigma(\mathrm{G})$ \\
\hline & Ambiente (A) & $\sigma($ erro $)+1,4788 \sigma\left(\mathrm{G}^{*} \mathrm{~A}\right)+\mathrm{Q}(\mathrm{A})$ \\
\hline & $\mathrm{G}^{*} \mathrm{~A}$ & $\sigma($ erro $)+1,4545 \sigma\left(\mathrm{G}^{*} \mathrm{~A}\right)$ \\
\hline \multirow{3}{*}{ II } & Genótipo (G) & $\sigma($ erro $)+2,2744 \sigma\left(\mathrm{G}^{*} \mathrm{~A}\right)+2,7593 \sigma(\mathrm{G})$ \\
\hline & Ambiente (A) & $\sigma($ erro $)+1,4788 \sigma\left(\mathrm{G}^{*} \mathrm{~A}\right)+\mathrm{Q}(\mathrm{A})$ \\
\hline & $\mathrm{G}^{*} \mathrm{~A}$ & $\sigma($ erro $)+1,4545 \sigma\left(\mathrm{G}^{*} \mathrm{~A}\right)$ \\
\hline \multirow{3}{*}{ III } & Genótipo (G) & $\sigma($ erro $)+1,9608 \sigma\left(\mathrm{G}^{*} \mathrm{~A}\right)+2,4510 \sigma(\mathrm{G})$ \\
\hline & Ambiente (A) & $\sigma($ erro $)+1,4545 \sigma\left(G^{*} \mathrm{~A}\right)+\mathrm{Q}(\mathrm{A})$ \\
\hline & $\mathrm{G}^{*} \mathrm{~A}$ & $\sigma($ erro $)+1,4545 \sigma\left(\mathrm{G}^{*} \mathrm{~A}\right)$ \\
\hline \multirow{3}{*}{ IV } & Genótipo (G) & $\sigma($ erro $)+2,3704 \sigma\left(\mathrm{G}^{*} \mathrm{~A}\right)+2,3704 \sigma(\mathrm{G})$ \\
\hline & Ambiente (A) & $\sigma($ erro $)+1,4545 \sigma\left(\mathrm{G}^{*} \mathrm{~A}\right)+\mathrm{Q}(\mathrm{A})$ \\
\hline & $\mathrm{G}^{*} \mathrm{~A}$ & $\sigma($ erro $)+1,4545 \sigma\left(\mathrm{G}^{*} \mathrm{~A}\right)$ \\
\hline
\end{tabular}

${ }^{(1) /}$ forma quadrática.

Ciência Rural, v.36, n.6, nov-dez, 2006. 
$2,2448 \mathrm{~s}\left(\mathrm{G}^{*} \mathrm{~A}\right)+\mathrm{Q}(\mathrm{A})$ e s $($ erro $)+2,238 \mathrm{~s}\left(\mathrm{G}^{*} \mathrm{~A}\right)$ fornece um teste de melhor aproximação em relação tanto ao tipo I, como ao tipo II e ao tipo III.

Tal fato ocorre porque a diferença entre 2,2448 e 2,238 (Tipo IV) é menor em valor absoluto que a diferença entre 2,1921 e 2,238 do tipo III, por exemplo. Foram realizadas todas as estimativas dos diferentes tipos de soma de quadrados, com suas respectivas esperanças matemáticas como advertência. De acordo ao discutido anteriormente, deve ser prudentemente escolhida a soma de quadrados tipo IV após o exame das esperanças matemáticas dos quadrados médios. As funções estimáveis do tipo IV aparecem em situações práticas nas quais ocorrem caselas vazias. Elas são construídas de tal forma que os coeficientes médios envolvendo as células são balanceados; fato que possibilita que as hipóteses resultantes sejam interpretáveis (COSTA, 2000). Portanto, havendo casela vazia, a interpretação da soma de quadrados tipo IV não pode ser feita sem antes examinar as funções estimáveis envolvidas, para saber qual delas está sendo gerada e testada.

Os dados inseridos na tabela 4, exclusivamente para os exemplos não balanceados com casela vazia, são vistos na literatura como os mais complexos dentre todos até agora comentados. Assim como no exemplo anterior, pode ser concluído que o tipo III, para o efeito do fator genótipo, é o tipo de soma de quadrados que melhor fornecerá uma aproximação para o teste, em comparação com as demais somas de quadrados. Esta escolha entre os diferentes tipos de soma de quadrados não depende da natureza do fator, ou seja, tanto faz o fator ser de natureza fixa ou aleatória. Por exemplo, se o fator genótipo fosse denominado como de efeito fixo, a soma de quadrados tipo III seria também ser a escolhida.

Geralmente, as hipóteses do tipo IV são iguais às do tipo III, porém, segundo IEMMA (1995), se existe caselas vazias e mais de dois níveis do fator, então, em geral, as hipóteses dos tipos III e IV são diferentes. Assim, se existe caselas vazias, as somas de quadrados do tipo IV podem não ser únicas, ou pior ainda, o pesquisador pode julgar estar testando uma hipótese quando na verdade as funções lineares que a definem nem mesmo são estimáveis.

Para FILHO (2002), com o desenvolvimento do proc mixed do SAS, a análise deste tipo de experimentos, tratada pela metodologia de modelos lineares mistos, tornou-se mais usual e segura.

\section{CONCLUSÕES}

Para extrair toda a informação de experimentos que possuam dados incompletos, deve ser avaliada qual a soma de quadrados que tem lógica em relação ao plano experimental. Na presença de caselas vazias, deve ser utilizado o proc mixed.

\section{REFERÊNCIAS}

AGUIAR, A.M. Uso do delineamento III com marcadores moleculares para análise genética da produção de grãos e seus componentes em milho. 2003. 126f. Tese (Doutorado em Agronomia) - Curso de Pós-graduação em Agronomia, Universidade de São Paulo.

BARBIN, D. Componentes de variância - Teoria e aplicações. 2.ed. Piracicaba: FEALQ, 1998. 120p.

CARDELLINO, R.A.; SIEWERDT, F. Utilização adequada e inadequada dos testes de comparação de médias. Revista da Sociedade Brasileira de Zootecnia, Viçosa, v.21, n.6, p.985995, 1992

CORNFIELD, J.; TUKEY, J.W. Average values of mean squares in factorials. Annals of Mathematical Statistics, Alexandria, v.27, p.907-949, 1956.

COSTA, J.R. et al. Obtenção das somas de quadrados tipo I, II e III em experimentos em parcelas subdivididas com testemunhas na parcela. Ciência Agrotécnica, Lavras, v.24, n.4, p.998$1007,2000$.

COSTA, S.C. Modelos lineares generalizados mistos para dados longitudinais. 2003. 107f. Tese (Doutorado em Agronomia) - Curso de Pós-graduação em Agronomia, Universidade de São Paulo.

FALCONER, D.S.; MACKAY, T.F.C. Introduction to quantitative genetics. 4.ed. England: Longman, 1996. 463p.

FILHO, J.A.C. Modelos lineares MISTOS: estruturas de matrizes de variâncias e covariâncias e seleção de modelos. 2002. 85f. Tese (Doutorado em Agronomia) - Curso de Pósgraduação em Agronomia, Universidade de São Paulo.

FILHO, J.A.C. Uma aplicação de modelos lineares mistos. Curitiba, setembro. 2003. Capturado em 15 mai. 2006. Online. Disponível na Internet http://www.est.ufpr.br/rt/jom03.pdf.

FREUND. R.J.; LITTELL, R.C. SAS for linear models. Cary, NC. Sas Institute, 1981. 229p.

GETTING Started with the SAS ${ }^{\circledR}$ Learning Edition. Cary, NC: SAS Institute, 2002. 81p.

GOODNIGHT, J.H. Tests of hypothesis in fixed effects linear models. Communicatiopn Statistical Theoritical Methodology, Ney York, v.9, n.2, p.167-180, 1980.

HOCKING, R.R. The analysis of linear models. Monterey: Brooks/Cole, 1985. 385p.

IEMMA, A.F. Que hipóteses estatísticas testamos através do SAS em presença de caselas vazias? Scientia Agricola, São Paulo, v.52, p.210-220, 1995. 
JUNIOR, C.L. de S. Componentes da variância genética e suas implicações no melhoramento vegetal. São Paulo: Piracicaba: FEALQ, 1989. 134p.

KEMPTON, R.A.; FOX, P.N. Statistical methods for plant variety evaluation. New York: Chapman \& Hall, 1997. 185p.

LITTELL, R.C. et al. SAS system for mixed models. Cary, NC. Sas Institute, 1996. 633p.

NESI, C.N. Métodos alternativos para realização de testes de hipóteses em delineamentos experimentais. 2002.

112f. Dissertação (Mestrado em Estatística e Experimentação Agronômica) - Curso de Pós-graduação em Agronomia, Universidade de São Paulo.

RAMALHO M.A.P. et al. Genética na agropecuária. 3.ed. São Paulo: UFLA, 2004. 472p.

RAMALHO, M.A.P. et al. Experimentação em genética e melhoramento de plantas. São Paulo: UFLA, 2000. 293p.
SEARLE S. et al. Variance components. New York: John Willey, 1992. 501p.

SEARLE, S.R. Linear models. New York: John Wiley \& Sons, 1971. 532p.

SILVA, R.G. Manual de procedimentos em análise por quadrados mínimos. São Paulo: FUNEP, 1993. 169p.

SPEED, F.M.; HOCKING, R.R. The use of the R(.) notation with un balanced data. American Statician, Washinght, v.28, n.1, p.30-33, 1976.

WECHSLER, F.S. Fatoriais fixos desbalanceados: uma análise mal compreendida. Pesquisa Agropecuária Brasileira, Brasília, v.33, n.03, p. 231-262, 1998.

WINER, B.J. Statistical principles in experimental design. 2.ed. New York: McGraw-Hill, 1971. 907p. 